\title{
Considerações acerca dos conceitos de condutores, isolantes e semicondutores nos livros de Ensino Médio sob um olhar da teoria de bandas de energia
}

Considerations on the concepts of conductors, insulators and semiconductors in High School books under a look at energy banding theory

\author{
E. S. Correia ${ }^{1 *}$ J. M. Dantas ${ }^{1,2} ;$ J. E. Andrade ${ }^{1,3}$ \\ ${ }^{1}$ Faculdade de Física/Instituto de Ciências Exatas/Programa Nacional de Mestrado Profisional em Ensino de Física, \\ Universidade Federal do Sul e Sudeste do Pará, 68507-590, Marabá - Pará, Brasil. \\ ${ }^{2}$ Faculdade de Computação e Engenharia Elétrica/Instituto de Geociências e Engenharias, Universidade Federal do \\ Sul e Sudeste do Pará, 68507-590, Marabá - Pará, Brasil. \\ ${ }^{3}$ Engenharia Mecânica/Instituto de Geociências e Engenharias, Universidade Federal do Sul e Sudeste do Pará, \\ 68507-590, Marabá - Pará, Brasil.
}

*evandrobio23@gmail.com

(Recebido em 06 de dezembro de 2016; aceito em 05 de janeiro de 2017)

\begin{abstract}
Este artigo apresenta uma discussão sobre os conceitos de condutor, isolante e semicondutor no Ensino Médio, considerando a teoria de Bandas de Energia na caracterização e distinção entre esses conceitos. Para isso, realizou-se um estudo bibliográfico acerca do tema e fez-se uma análise de alguns livros didáticos utilizados atualmente no Ensino Médio de acordo com o Programa Nacional do Livro Didático, 2015 (PNLD 2015). Dessa forma, percebeu-se que a maioria dos livros analisados não faz uma abordagem do ponto de vista da Física Quântica para explicação desses conceitos, o que motiva a uma reestruturação dos livros de Física no Ensino Médio.

Palavras-chave: Condutores e isolantes, Semicondutores, Livro didático.
\end{abstract}

This paper discusses the concept of conductor, insulating and semiconductor materiais in High School considering the Theory of Energy Band in order to the characterize and distinguish them. For this purpose, a bibliographic study was carried out and an analysis was made based on High School didactic books according to the Programa Nacional do Livro Didático, 2015 (PNLD 2015). In this way, it was noticed that most of the books analyzed do not consider an approach from the point of view of Quantum Physics to explain these concepts, which motivates a restructuring of the Physics books adopted in High School level.

Keywords: Conductors and insulating, Semiconductors, Didactic book.

\section{INTRODUÇÃO}

Atualmente vivemos cercados por tecnologias que utilizamos nas mais diversas situações do cotidiano. Essas inovações têm facilitado nossos afazeres diários, garantindo uma melhor qualidade de vida e otimização do tempo. Podemos citar, por exemplo, a energia elétrica e todos os aparelhos eletroeletrônicos que funcionam a partir de uma corrente elétrica: TV, rádio, computador, celular, etc. A maioria dessas inovações surgiu à luz da Física, graças à descoberta de alguns materiais que merecem destaque por tamanha importância na evolução tecnológica da humanidade: materiais condutores de eletricidade, isolantes ou dielétricos e semicondutores.

No entanto, você já se perguntou qual a diferença entre esses materiais? Obviamente, a resposta é "trivial" em termos de condutores e isolantes, no sentido de que eles estão relacionados com o nosso cotidiano desde a infância ("Cuidado menino, esse fio conduz eletricidade e você pode tomar um choque!") e ao longo da educação básica, nas disciplinas de Ciências e Física, principalmente. Porém, não se pode dizer o mesmo dos semicondutores, uma vez que o entendimento do fenômeno mais preciso só ocorreu com o advento da Física Quântica. 
Nesse sentido, o presente artigo faz algumas considerações acerca dos conceitos de condutores, isolantes e semicondutores apresentados nos livros didáticos do Ensino Médio sob um "olhar" da Física Quântica, abordando, principalmente, a Teoria de Bandas de Energia.

$\mathrm{O}$ trato desse tema é relevante ao ponto que a própria Lei de Diretrizes e Bases da Educação Brasileira (LDB) [1] em seu artigo 35, mais especificamente no inciso IV, estabelece como finalidade do Ensino Médio: a compreensão dos fundamentos científico-tecnológicos dos processos produtivos, relacionando a teoria com a prática, no ensino de cada disciplina. Dessa forma, os conceitos de condutor, isolante e semicondutor representam um pré-requisito para que os discentes possam compreender as inúmeras tecnologias da atualidade, considerando, é claro, os seus aspectos mais atuais, incorporados a partir das novas teorias.

Concomitante, as Orientações Curriculares para o Ensino Médio (2008) [2] estabelecem que os saberes escolares devem proporcionar aos alunos a aquisição de elementos de compreensão e/ou manuseio de aparatos tecnológicos, de máquinas e processos de produção industrial ampliando a compreensão do mundo em que vivem.

Sendo assim, a Física, como uma disciplina científica e sistematizada a partir de seus conceitos, princípios e leis, assim como as demais ciências, está em constante reformulação e ampliação. Portanto é imprescindível que a Física Escolar também o seja. Pensando nisso, destaca-se o "papel" do livro didático, que é muito importante na compreensão dos conceitos de condutor isolante e semicondutor, uma vez que o mesmo é utilizado como uma das principais ferramentas de ensino pelos professores de Física.

De modo geral, o presente artigo objetiva apresentar uma análise sucinta de alguns livros didáticos no que se refere aos conceitos de condutor, isolante e semicondutor levando em consideração a abordagem da teoria de Bandas de Energia.

Para compreendermos a teoria de bandas de energia pode-se tomar como exemplo um átomo de cobre cuja distribuição eletrônica é a seguinte: $1 \mathrm{~s}^{2} 2 \mathrm{~s}^{2} 2 \mathrm{p}^{6} 3 \mathrm{~s}^{2} 3 \mathrm{p}^{6} 3 \mathrm{~d}^{10} 4 \mathrm{~s}^{1}$. Evidentemente, a subcamada de energia $4 \mathrm{~s}^{1}$ (camada de valência do átomo de cobre) comporta no máximo dois elétrons, mas ela apresenta apenas um. Portanto, quando aproxima-se dois átomos de cobre, devese considerar que "(...) existe uma onda de matéria associada ao elétron. Se o elétron fosse apenas uma partícula, como suponha a física clássica, a energia do elétron poderia ter qualquer valor, mesmo quando estivesse confinado em uma armadilha." [3].

As ondas de matéria associadas aos elétrons dos dois átomos se superpõem de modo que tem-se o número de camadas energéticas (níveis de energia) duplicadas, seguindo o Princípio da Exclusão de Pauli. De acordo com esse princípio, "não podem existir dois elétrons com os mesmos números quânticos. Em outras palavras, cada orbital pode ser ocupado apenas por um elétron." [4]. Evidentemente, trata-se de elétrons de uma mesmo material.

O mesmo acontece em um sólido cristalino com $\mathrm{N}$ átomos em sua estrutura, onde os níveis de energia de cada átomo serão subdivididos em $\mathrm{N}$ níveis formando, assim, as bandas de energia, que são separadas por bandas proibidas (band gap). As bandas proibidas representam "níveis de energia que nenhum elétron pode ocupar." [3].

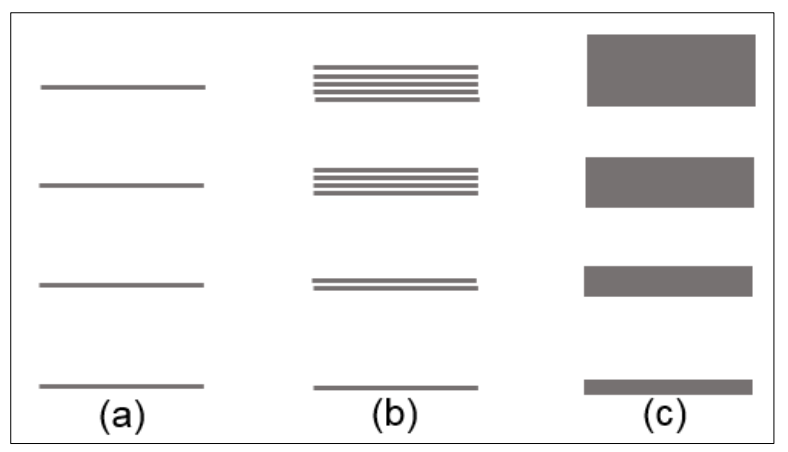

Figura 1: Ilustração da formação de bandas de energia nos sólidos.

Conforme a Figura 1, tem-se que (a) representa a distribuição eletrônica de um átomo; em (b) a distribuição em vários átomos; em (c) a formação de bandas de energia separadas por bandas proibidas. 
Segundo Kittel, [4] "a questão importante para a condutividade elétrica é como os elétrons respondem a um campo elétrico aplicado." Nesse caso, é importante frisar o conceito de banda proibida, no sentido de que ela representa o potencial energético a ser superado pelos elétrons quando esses são submetido a um determinado campo elétrico, aumentando, assim, sua energia cinética. Se a energia adquirida pelos elétrons for maior que a energia da banda proibida esses elétrons passam a ocupar uma banda de energia superior, banda de condução, caracterizando o material como condutor elétrico.

Portanto, partindo da ideia de bandas de energia pode-se definir condutores, isolantes e semicondutores conforme Kittel [4] afirma:

O cristal se comporta como isolante se todas as bandas de energia permitidas estão totalmente cheias ou totalmente vazias, porque nesse caso nenhum elétron pode se mover em resposta à aplicação de um campo elétrico. O cristal se comporta como metal se uma ou mais bandas está parcialmente cheia, com 10 a $90 \%$ da capacidade, digamos. O cristal se comporta como um semicondutor ou semimetal se uma ou mais bandas está quase cheia ou quase vazia.

Pode-se, agora, analisar os condutores, isolantes e semicondutores a partir da figura 2.

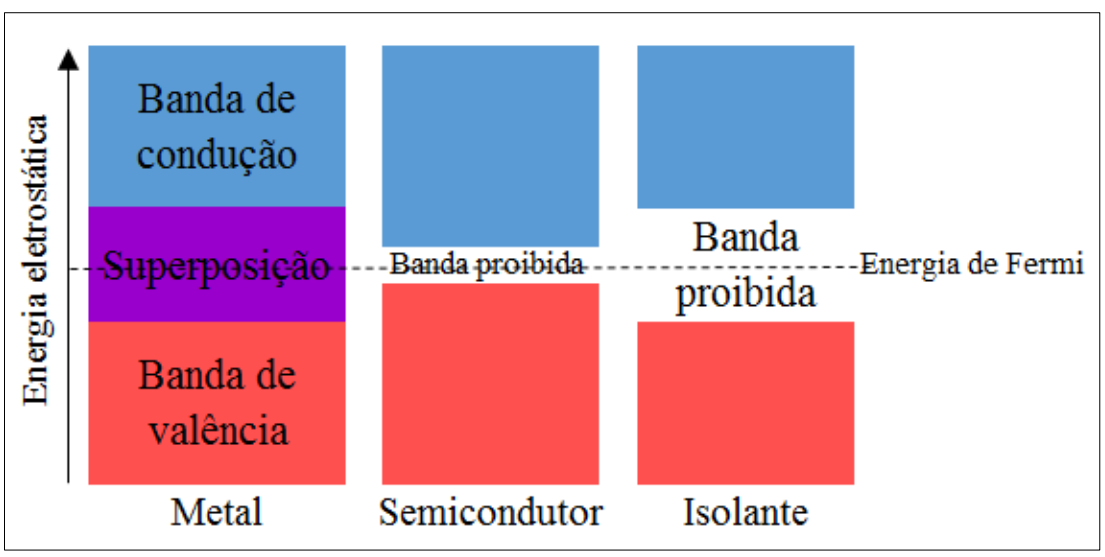

Figura 2: Representação das bandas de energia em metais, semicondutores e isolantes.

Conforme a Figura 2, nos materiais condutores as bandas de valência e de condução se superpõem (retângulo violeta), de modo que sempre vão existir elétrons na banda de condução, tornando-os bons condutores de eletricidade. Em outras palavras, em um condutor, ao menos uma banda de energia encontra-se parcialmente preenchida. [5].

Halliday et al. [3] também define que, em um condutor, "o nível de energia mais alto ocupado pelos elétrons está no meio de uma banda de energia permitida." Halliday et al. [3] especifica que o nível de Fermi representa o nível mais alto ocupado da banda na temperatura de zero absoluto (T $=0)$ e a energia correspondente a esse nível é chamada de energia de Fermi.

Nos materiais isolantes a banda de valência está completamente cheia e, de acordo com Princípio da Exclusão de Pauli, os elétrons não podem ocupar um nível energético mais alto que esteja completamente preenchido; nos isolantes, a banda proibida apresenta uma intensidade muito alta, nesse caso, para os elétrons "saltarem" da banda de valência para a banda de condução é necessário fornecer uma quantidade de energia elevada ao material, provocando a quebra da rigidez dielétrica. Nos isolantes, a energia dos elétrons deve ser muito grande para que possa haver uma corrente elétrica.

Os materiais semicondutores apresentam uma estrutura de banda de energia semelhante à dos isolantes diferindo na intensidade ou largura da banda proibida, pois, nos semicondutores a energia de gap (Eg) é bem menor (inferior a $2 \mathrm{eV}$ ), ou seja:

(...) a distância $\mathrm{E}_{\mathrm{g}}$ entre o nível mais alto da última banda ocupada (a banda de valência) e o nível mais baixo da primeira banda desocupada (a banda de condução) é muito menor que nos isolantes. Assim, por exemplo, o silício $\left(\mathrm{E}_{\mathrm{g}}=\right.$ $1,1 \mathrm{eV})$ é um semicondutor, enquanto o diamante $\left(\mathrm{E}_{\mathrm{g}}=5,5 \mathrm{eV}\right)$ é um isolante. No silício, ao contrário do que acontece no diamante, existe uma probabilidade significativa de que a agitação térmica faça um elétron passar da banda de valência para a banda de condução. [3]. 
De acordo com Ashcroft e Mermin (2011) [5] "Sólidos isolantes em T = 0, mas cujo gap de energia é de tal tamanho que a excitação térmica pode levar à condutividade observável em temperaturas abaixo do ponto de fusão, são conhecidos como semicondutores".

Um fato relevante em relação aos semicondutores ocorre quando os elétrons de sua banda de valência são termicamente excitados para a banda de condução deixando buracos na banda de valência numa proporção que varia com o inverso da temperatura $(1 / \mathrm{T})$, ou seja, o aumento da temperatura provoca um aumento da condutividade do semicondutor, apresentando um comportamento elétrico diferente dos metais (condutores) que, por sua vez, diminuem sua condutividade com o aumento da temperatura. Sendo assim, os semicondutores têm sua resistência elétrica diminuída com o aumento da temperatura [5], característica muito importante para o desenvolvimento tecnológico (fabricação de transistores, comutadores, diodos, células fotovoltaicas, detectores e termistores).

\section{MATERIAL E MÉTODOS}

Para o desenvolvimento deste artigo foram analisados os conceitos de condutor, isolante e semicondutor apresentados em oito livros didáticos de Física do terceiro ano do Ensino Médio, de acordo com o PNLD de 2015. Realizou-se, também, um estudo bibliográfico relacionado aos conceitos acima citados.

O PNLD 2015 foi norteador, no sentido de estabelecer os critérios de avaliação, ou seja, os parâmetros utilizados na classificação das obras inscritas no Plano.

Chama-se a atenção aqui para os seguintes critérios estabelecidos pelo PNLD 2015 [6]: "Correção e atualização de conceitos, informações e procedimentos", que estabelece o respeito às conquistas científicas da área da Física incluindo as novas teorias, conceitos e princípios que vão surgindo ao longo do desenvolvimento científico; especificamente em relação ao componente curricular Física, o PNLD 2015 observou se a respectiva obra:

Tratou de forma adequada e pertinente, considerando os diversos estudos presentes na literatura atual da área, tópicos usualmente classificados como de Física Moderna e Contemporânea e que sejam considerados importantes ou mesmo imprescindíveis para o exercício da cidadania ativa, crítica e transformadora, bem como para a inserção ativa, crítica e transformadora no mundo do trabalho. [6].

O PNLD 2015 aprovou um total de 14 obras didáticas relacionadas a Física, dentre as quais pôde-se analisar apenas oito, conforme a tabela abaixo. Os demais livros não foram analisados por falta de acesso. 
Tabela 1: Relação dos livros analisados.

\begin{tabular}{l|l|c|c}
\hline \multicolumn{1}{c|}{ Livro } & \multicolumn{1}{|c|}{ Autores } & Editora & Edição/Ano \\
\hline Física 3 (I) & $\begin{array}{l}\text { José Roberto C. Piqueira; } \\
\text { Wilson Carron; José } \\
\text { Osvaldo de S. Guimarães }\end{array}$ & Ática & $1^{\mathrm{a} / 2013}$ \\
\hline Física 3 (II) & $\begin{array}{l}\text { Ricardo Helou Doca; } \\
\text { Newton Villas Bôas; } \\
\text { Gualter José Biscuola }\end{array}$ & Saraiva & $2^{\mathrm{a} / 2013}$ \\
\hline Física (III) & $\begin{array}{l}\text { Alysson Ramos Artuso; } \\
\text { Marlon Wrublewski }\end{array}$ & Positivo & $1^{\mathrm{a} / 2013}$ \\
\hline Física Aula por Aula (IV) & $\begin{array}{l}\text { Claudio Xavier; Benigno } \\
\text { Barreto }\end{array}$ & FTD & $2^{\mathrm{a} / 2013}$ \\
\hline Física Ciência e Tecnologia (V) & $\begin{array}{l}\text { Carlos Magno A. Torres; } \\
\text { Nicolau Gilberto Ferraro; } \\
\text { Paulo Antonio de T. Soares; } \\
\text { Paulo Cesar Martins } \\
\text { Penteado }\end{array}$ & Moderna & $3^{\mathrm{a} / 2013}$ \\
\hline Física Contexto \& Aplicações (VI) & $\begin{array}{l}\text { Antônio Máximo; } \\
\text { Beatriz Alvarenga }\end{array}$ & Scipione & $1^{\mathrm{a} / 2013}$ \\
\hline Física para o Ensino Médio (VII) & $\begin{array}{l}\text { Luiz Felipe Fuke; } \\
\text { Kazuhito Yamamoto }\end{array}$ & Saraiva & $3^{\mathrm{a} / 2013}$ \\
\hline Ser Protagonista Física (VIII) & Angelo Stefanovits & $\begin{array}{c}\text { Edições } \\
\text { SM }\end{array}$ & $2^{\mathrm{a} / 2013}$ \\
\hline
\end{tabular}

$\mathrm{Na}$ análise dos livros acima citados foram realizadas leituras dos tópicos relacionados à Eletricidade e à Física Quântica, afim de se verificar a abordagem dada aos conceitos de condutor, isolante e semicondutor. Nesse sentido, os dados coletados foram organizados em uma tabela cujas colunas foram dispostas de modo a responder aos seguintes questionamentos: Qual o título do livro? Qual o autor? O livro apresenta os conceitos de condutor e isolante segundo a Física Clássica? O livro apresenta o conceito de semicondutor do ponto de vista da Física Clássica? O livro faz uma abordagem dos conceitos de condutor e isolante segundo a teoria de Bandas de Energia? O livro aborda o conceito de semicondutor segundo a teoria de Bandas de Energia? Entrelaçado às respectivas respostas e com o respaldo da teoria de Bandas de Energia, foram feitas as análises dos livros conforme a próxima seção.

\section{RESULTADOS E DISCUSSÃO}

De uma maneira geral, todos os livros listados na Tabela 1 apresentam os conceitos de condutores e isolantes de maneira satisfatória, sem o aparato da teoria de bandas de energia. Porém, nem todos abordam o conceito de semicondutores.

Em I e IV, não é feita nenhuma referência aos semicondutores e/ou a teoria de bandas de energia.

No livro II são listados alguns exemplos de materiais que diminuem a resistividade com o aumento da temperatura (característica dos semicondutores): "Na grafite, no silício e no germânio, a resistividade diminui quando a temperatura aumenta. (...) Assim aumenta a população de elétrons livres e o material torna-se um condutor melhor do que era." [7]. O conceito de semicondutor não é apresentado no livro, porém, o autor sugere uma pesquisa sobre o tema, destacando algumas aplicações tecnológicas e a teoria de bandas. Dessa forma, percebe-se que tanto o aluno como o professor podem explorar os conceitos em outras fontes e, possivelmente realizarem um debate em sala.

Dentre os livros analisados, cabe destacar o III, que por sua vez, apresenta um aparato conceitual mais bem elaborado sobre os condutores, isolantes e semicondutores a partir da teoria de bandas de energia. No texto são destacados os conceitos, bem como, ilustrações relacionadas que possibilitam 
um bom entendimento da teoria de bandas e das diferenças entre os condutores, isolantes e semicondutores, conforme segue:

Atomicamente falando, a condução elétrica ocorre quando os elétrons da banda de valência de um átomo são promovidos por um estímulo energético externo para uma banda mais energética, denominada banda de condução, na qual os elétrons têm liberdade para se movimentarem de forma mais ordenada, caracterizando a condução elétrica. [8].

Além disso, ao abordar o conteúdo de resistência elétrica, o autor ainda compara a resistividade de alguns desses materiais. É evidente que os alunos teriam uma melhor compreensão desses conceitos após estudarem o capítulo relacionado à Física Quântica que trata do princípio da incerteza, ondas de matéria, função de onda, Princípio de Exclusão de Pauli, etc.

Em V, o conceito de semicondutor é apresentado de maneira bem resumida, sem explorar a teoria de bandas de energia, mostrando apenas a sua importância na produção dos transistores: "A maioria dos circuitos elétricos modernos usa elementos conhecidos como semicondutores, como silício ou germânio. (...) Os semicondutores são usados nos transistores, que são fundamentais nos circuitos de computadores, nos amplificadores etc." [9].

No livro VI, faz-se uma abordagem sobre os semicondutores relacionando a sua resistência elétrica com a variação de temperatura, mas não explica a diferença entre condutores, isolantes e semicondutores utilizando a teoria de bandas; relaciona a fabricação dos transistores a partir dos semicondutores destacando sua importância tecnológica; aborda de maneira sucinta os semicondutores tipo $n$ e $p$ oriundos de processos de dopagem: (...) Assim, adicionando-se uma pequena quantidade de arsênio a uma amostra de silício, obtém-se um condutor elétrico semelhante a um metal (...). Dizemos que um semicondutor como esse é do tipo n (condução feita por cargas negativas). [10].

O livro VII apresenta uma noção geral dos semicondutores e faz um pequeno comentário sobre o processo de dopagem, que é muito importante na engenharia de materiais uma vez que influencia nas propriedades físicas do material (condutibilidade, maleabilidade, resistividade, etc.). Porém, a teoria de banda de energia não é abordada pelos autores.

No livro VIII, destaca-se uma das principais características dos semicondutores (apresenta resistência elétrica intermediária em relação a condutores e isolantes) e o relaciona com a fabricação de transistores, destacando os avanços na eletrônica. Mas também não utiliza a teoria de bandas para distinguir os semicondutores dos isolantes e metais.

Em suma, pode se afirmar que apenas 12,5\% dos livros analisados apresentam os conceitos de condutores, isolantes e semicondutores sob uma perspectiva da teoria de bandas de energia. Esse percentual é muito pequeno considerando a proposta do PNLD em que os livros didáticos deve incorporar os novos conceitos que vão surgindo com os avanços científicos. Ademais, condutores isolantes e semicondutores são assuntos pertinentes aos avanços tecnológicos que ocorrem a cada dia e impactam o nosso cotidiano de maneira direta. Um exemplo são os celulares, cada vez mais modernos, com uma capacidade de armazenamento e processamento muito grande graças a tecnologias como transistores que, por sua vez, só foram concebidas a partir dos semicondutores.

\section{CONCLUSÃO}

Considerando que os livros didáticos do ensino médio são reformulados a cada três anos, de acordo com o PNLD, é recomendável que todas as editoras, bem como os avaliadores especialistas e os professores, fiquem atentos na elaboração, análise e escolha dos livros de Física, de modo a contemplar, no capítulo que trata da Física Quântica, a teoria de bandas de energia, condutores, isolantes e semicondutores.

Por mais que os alunos tenham contato com esses conceitos nos primeiros capítulos do livro de Física do $3^{\circ}$ ano do ensino médio, abordagem Clássica, a discussão sobre o assunto devem ser retomada após o estudo da Física Quântica, pois dessa forma, o conhecimento será mais significativo a partir das noções de ondas de matéria, princípio da incerteza, função de onda, Princípio de Exclusão de Pauli, etc.

Portanto, no que se refere aos livros didáticos de Física, sugere-se que todos contemplem os conceitos de condutor, isolante e semicondutor sob um olhar da teoria de bandas de energia, que 
por sua vez é mais atualizada e rebuscada, garantindo uma compreensão mais adequada dos semicondutores e das tecnologias a eles relacionadas como exemplo, os transistores, que revolucionaram a eletrônica.

\section{REFERÊNCIAS BIBLIOGRÁFICAS}

1. Brasil. Lei n $^{\circ}$ 9.394, de 20 dezembro de 1996: Estabelece as Leis de Diretrizes e Bases da Educcação Nacional..

2. Brasil. Ciências da Natureza, Matemática e suas Tecnologias: orientações curriculares para o Ensino Médio Brasilia; 2008.

3. Halliday D, Resnick R, Walker J. Fundamentos de Física: óptica e física moderna. 9th ed. Rio de Janeiro: LTC; 2012.

4. Kittel C. Introdução á física do estado sólido Rio de Janeiro: LTC; 2006.

5. Ascroft NW, Mermin ND. Física do Estado Sólido São Paulo: Cergage Learning; 2011.

6. Brasil. Guia de livros didáticos: PNLD 2015: física: ensino médio Brasilia: Secretaria de Educação Basica; 2014.

7. Biscuola GJ, Doca RH, Bôas NV. Física 3. 2nd ed. São Paulo: Saraiva; 2013.

8. Artuso AR, Wrublewski M. Física Curitiba: Positivo; 2013.

9. Torres CMA, Farraro NG, Soares PAdT, Penteado PCM. Física: ciência e tecnologia: eletromagnetismo e física moderna. 3rd ed. São Paulo: Moderna; 2013.

10. Luz AMRd, Álvares BA. Física contexto e aplicações. 1st ed. São Paulo: Scipione; 2013. 